\title{
Pre-Warning System for Sudden Events in Public Based on Pedestrian Behavior's Understanding from Surveillance Video
}

\author{
Yuan-zhi LIANG ${ }^{1 *}$ \\ ${ }^{1}$ Lanzhou University, China
}

Keywords: K-medoids, Wavelet neural network, Surveillance video processing.

\begin{abstract}
Surveillance video records the situation of pedestrians in public. If some sudden event such as a robbery or someone gravely ill, guards or managers can get this by watching and understanding pedestrians behaviors from surveillance video in time. In this paper, we propose a system to warning the guard that there are something abnormal in public by analyzing people action from the video. This system contains three subsystem. Firstly, we adapt a wavelet neural network (WNN) for predicting the number of pedestrians. It sends alarm when the amount of people become excessively large. Secondly, we record the direction of every pedestrians and if most people go to the same direction instead of go to all the direction randomly, such situation should be treated with caution. Thirdly, the k-medoids clustering algorithm is used to calculate cluster center and cluster size of current pedestrian for describing distribution situation and give an alarm if the distribution becoming exceptional. Finally, we use a pedestrian database to exam our system.
\end{abstract}

\section{Introduction}

With development of society and economy, the deployment of video surveillance in public space such as stations, squares has become normalcy. Surveillance video processing and pedestrian behaviors understanding draw a lot of attention for its increasing demand. The most popular issue in this area is crowd counting. Pedestrian counting in public places has a wide spectrum of applications in crowd control especially in people risk behaviors control in public. While, take the difficulty of pedestrian detection counting and tracking into consideration, in some applications, crowd counting on a train platform, estimating to a global count for the whole scene is sufficient. Most state-of-the-art methods are regression based and the goal is to learn a mapping between low-level feature and crowd counts. [3, 4, 5] Some deep-learning based method also be proposed for counting crowd precisely. [2] However, in the area of behaviors control or sudden event early-warning, counting result of pedestrian do not have a very high requirements so we adapt a simple method for pedestrian counting. In addition, pedestrian behaviors understanding is also extensively studied [1]. There are many challenging task in this issue such as walking path prediction $[9,10]$, traffic flowing segmentation $[11,12,13]$, crowd or particular target segmentation [14], and abnormal event detection. $[15,16]$

To our knowledge, a pre-warning system in public has seldom been researched which is also important to the reality. While, inevitably, some sudden events occur in public sometime. People may meet something like robbery, fight, squabble, fire, or even stampede accident. In order to avoid such event, the managers or guards should see the surveillance video constantly. In this paper, we propose a system to warning the guard that there are something abnormal in public by analyzing people action from the video. The system contains three subsystem and each subsystem focus on a kind of crowd 
behaviors. Not only the current pedestrian behaviors, but also predicting pedestrian behaviors are taken into account. Three subsystems are independent but cooperate with others. Some abnormal situations are efficiently detected and warning or early-warning signals are given out exactly.

\section{Proposed System Model}

The proposed system can be described by the follow block diagram Figure 1 and follow chart Figure 2. Obviously, the system contains three parts: pedestrian number forecast model by WNN, pedestrian distribution situation describe model by k-medoids clustering algorithm and traveling direction calculating model.

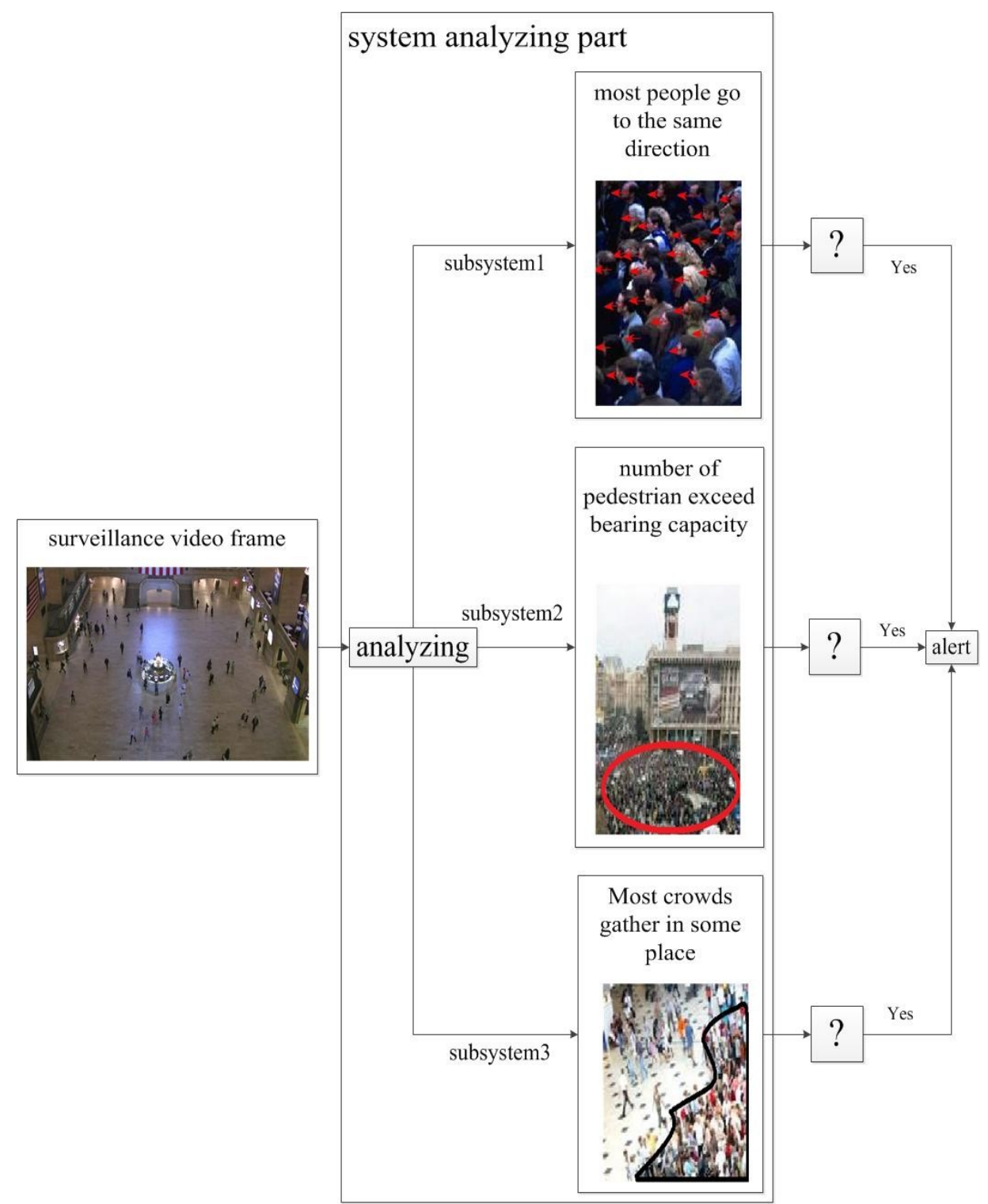

Figure 1: block diagram for proposed pre-warning system 


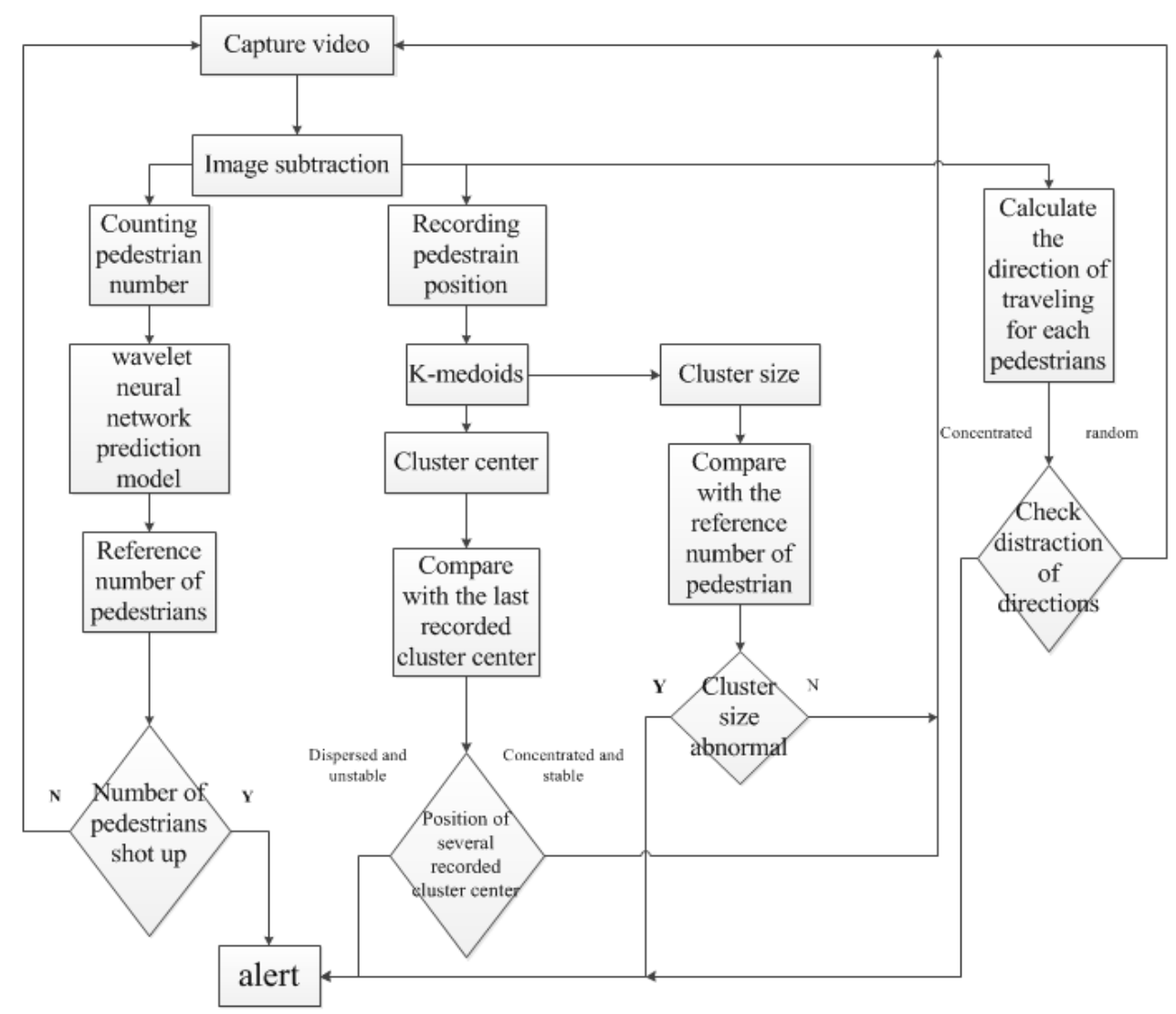

Figure 2: flow chart of proposed method

\section{Forecast Model by WNN}

First, the number of pedestrian could be counted by extracting some adjacent frames from video and subtracting two images to get an image contains pedestrian information like Figure 3. It is easy to get the number of pedestrian by the subtraction image.
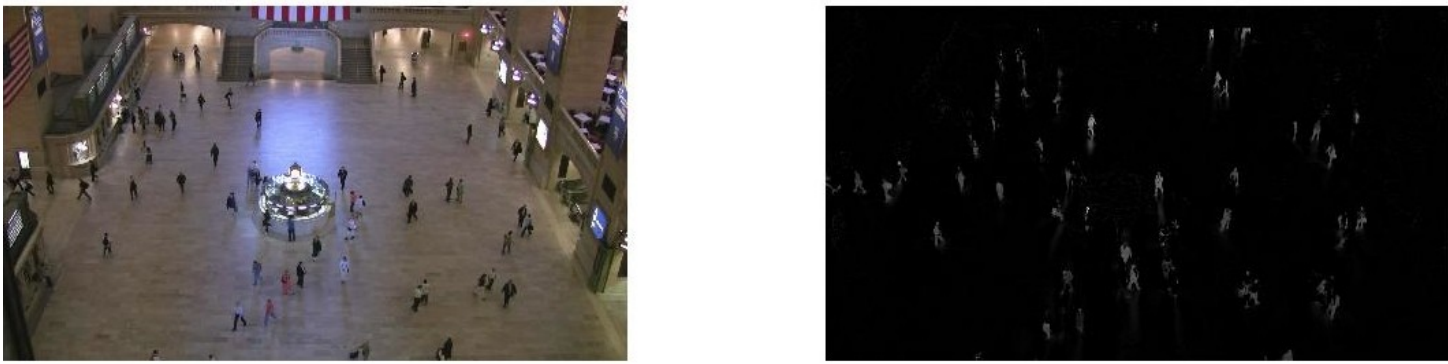

Figure 3: Extracted frame and subtraction image

Then applying a series of pedestrian number data into the WNN. WNN is a combination of classic sigmoid neural network and the wavelet analysis [6]. WNN, as a model, can be separated in two parts: model selection and variable significance testing [6]. WNN has three layers in structure and there are wavelet activation functions in hidden layer instead of other traditional activation functions. The structure of a WNN, which showed in follow Figure 4, with output $F(x)$, inputs $p\left\{x_{1}, x_{2}, \ldots \ldots x_{p}\right\}$ and $\mathrm{m}$ number of mother wavelet [7]. 


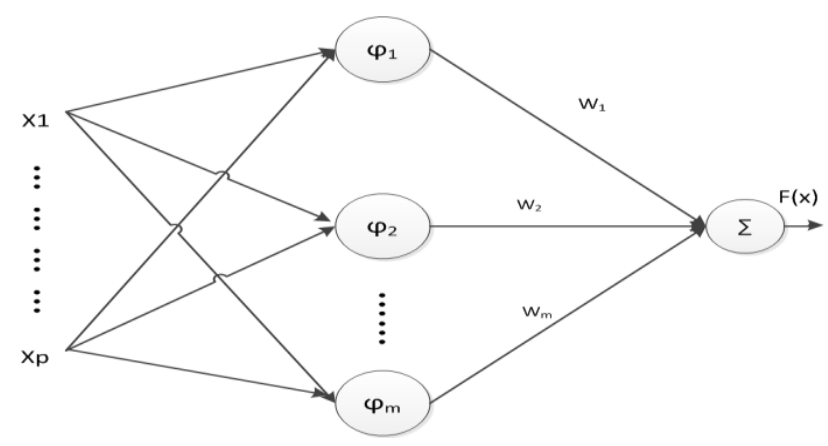

Figure 4: WNN model.

The output of a standard form WNN is given by: [7]

$$
F(x)=\sum_{i=1}^{M} w_{i} \Psi_{i}(x)=\sum_{i=1}^{M} w_{i}\left|a_{i}\right|^{-1 / 2} \Psi\left(\left(x-b_{i}\right) / a_{i}\right)
$$

In $i$ th unit of the hidden layer, $\Psi i$ is the wavelet activation function and $w_{i}$ is the wright connecting the $i$ th unit of the hidden layer to the output layer unit. Note that for the n-dimensional input space, the multivariate wavelet basis function can be calculated by the tensor product of $\mathrm{n}$ single wavelet basis functions as follows. Back-propagation used in training the WNN. [7]

$$
\Psi(x)=\prod n \quad i=1 \Psi(x i)
$$

By predicting the number of pedestrian, it is available to monitoring the number and the change rate in time. If the prediction number is far from current number (that means a rapid rate of number changing) or forecast number larger than the bearing capacity of current environment, a pre-warning signal should be given to the manager or guard for dealing with the abnormal event.

\section{Pedestrian Distribution Describe Model by K-medoids Clustering Algorithm}

The k-medoids algorithm is a clustering algorithm related to the k-means algorithm and the medoidshift algorithm. It is more robust to noise and outliners as compared to k-means because it minimizes a sum of pairwise dissimilarities instead of a sum of squared Euclidean distances and this paper applies the Voronoi iteration method [8] which works as follows:

1. Select initial medoids

2. Iterate while the cost decreases:

2.1 In each cluster, make the point that minimizes the sum of distances within the cluster the medoids

2.2 Reassign each point to the cluster defined by the closest medoids determined in the previous step.

By k-medoids clustering algorithm, the pedestrians of different positions in image could be clustered and divided into several groups. Every group has a kind of cluster center and cluster size. If the number of people in some groups are preponderantly apparently or the position of cluster center never changes in a couple of frames from the video, It must be something happened and attracted attention of most people which is never supposed to be occurred in this public space. And this status should be treated with caution as well. 


\section{Traveling Direction Calculating Model}

A comparing between two frames can provide the traveling direction of each pedestrian in the surveillance video as follow Figure 5.

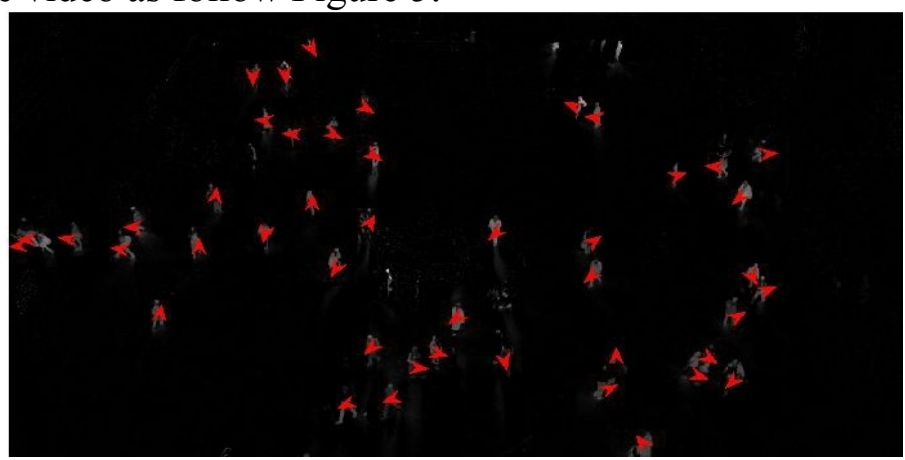

Figure 5: determine the traveling direction of pedestrians

If the traveling directions of most pedestrian tend to be the same direction, it may means that something attracted people to particular location or something horrified the crowd and forced people run away from the incident. In this case, an alarm to the guard is necessary.

\section{Experiments}

\section{The WNN model test}

In our experiments, the WNN has 2 input mode, 2 output node and 8 node in hidden layer. The Figure 6 shows relationship between test data and prediction. Horizontal axis shows the label of a consecutive sequence of frames and vertical axis shows the number of people. Model shows a good performance of forecast rate (maximum error rate: $8.9 \%$ ) and play an important role in the pre-warning system. From the result, prediction is always less than the test data, because if someone stand still or just make a small gesture, the subtraction image could not show them off and the model is unable to process these missing data. However, as shown in Figure 6, this kind of people just are a tiny proportion of the total number and do not much affect the prediction.

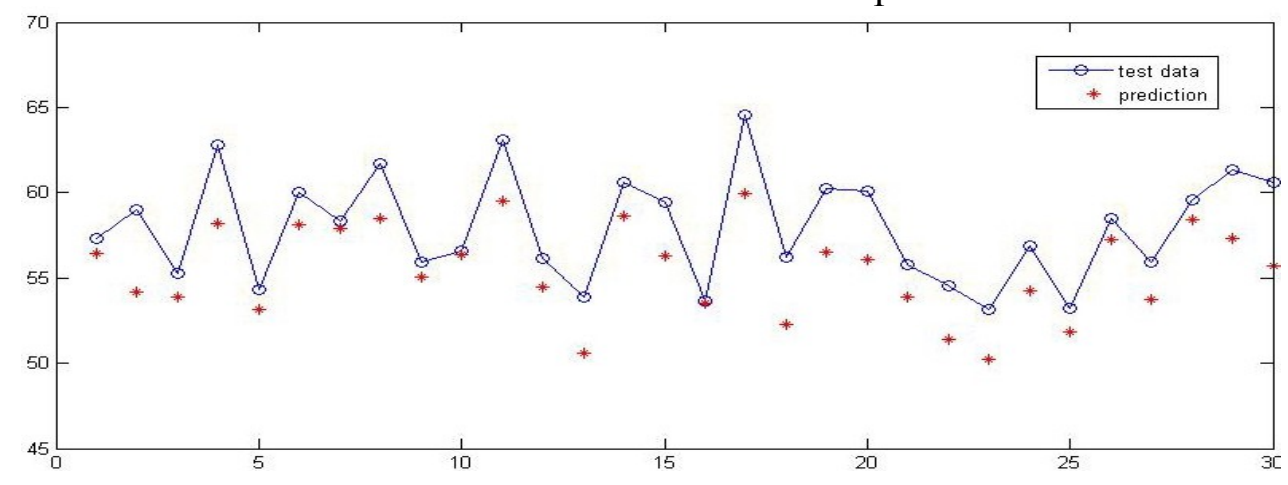

Figure 6: WNN test result.

\section{System Test}

In order to exam our system in existing dataset, we set a smaller limit number than the true bearing capacity. During the test, the system raised the alarm efficiently and met anticipate effect. Some frames are showed in follow Figure 7 which makes the system sending warning. 


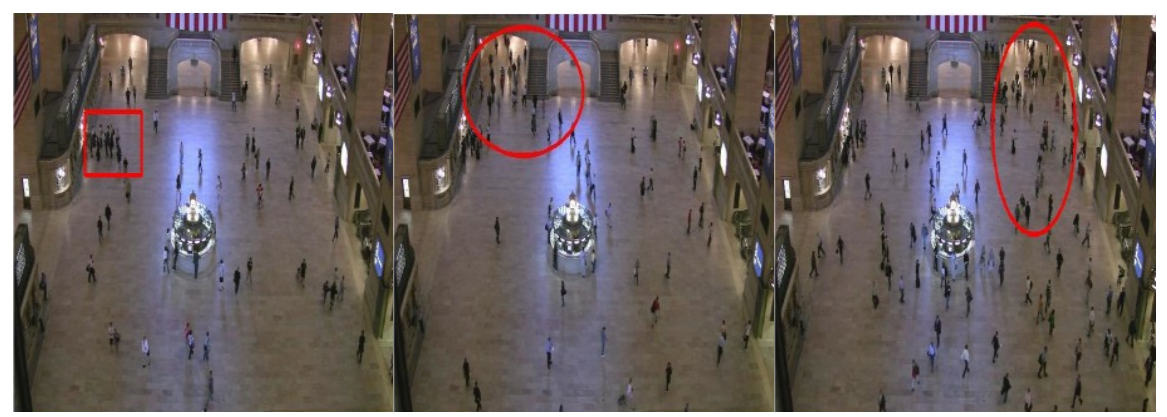

Figure 7: In the first image (left side), people gather in front of a store and the system finds that a cluster size is obviously bigger than the others after k-medoids algorithm. In the second and third image (middle and right side), the number of pedestrian in the lobby sharply increase and makes the system make alert.

\section{Conclusion}

In this paper, a pre-warning system was proposed. This system has three subsystem and each system have a kind of ability to monitor the pedestrian or analysis people behaviors. The system not only responds to the current pedestrian number and crowd behaviors, but also forecasts what the pedestrian tend to be. This method provides a new way for dealing with sudden event in public and makes the managers and guard having a quicker reacting to these incidents.

\section{Acknowledgements}

This work is supported by research funds for National Training Programs of innovation and Entreprleneurship for Undergraduates.(LanZhou University, project number: 201610730084)

\section{References}

[1] Shuai Yi, Hongsheng Li, and Xiaogang Wang. Understanding Pedestrian Behaviors from Stationary Crowd Groups. In Proc. of CVPR, IEEE, 2015.

[2] A. B. Chan, Z. S. Liang, and N. Vasconcelos. Privacy preserving crowd monitoring: Counting people without people models or tracking. In Proc. of CVPR, IEEE, 2008.

[3] K. Chen, S. Gong, T. Xiang, Q. Mary, and C. C. Loy. Cumulative attribute space for age and crowd density estimation. In Proc. of CVPR, 2013

[4] K. Chen, C. C. Loy, S. Gong, and T. Xiang. Feature mining for localised crowd counting. In In Proc. of BMVC, 2012.

[5] C. C. Loy, S. Gong, and T. Xiang. From semi-supervised to transfer counting of crowds. In Proc. of ICCV, IEEE, 2013.

[6] Antonios K.Alexandridis, Achilleas D.Zapranis. Wavelet neural networks: A practical guide. Preprint Published in Neural Networks, 42, pp. 1-27, 2013

[7] Mohammed Awad. Chaotic Time series Prediction using Wavelet Neural Network. Journal of Artificial Intelligence: Theory and Application (Vol.1-2010/Iss.3)

Awad / Chaotic Time series Prediction using Wavelet Neural Network/ pp. 73-80

[8] https://en.wikipedia.org/wiki/K-medoids 
[9] G. Antonini, S. V. Martinez, M. Bierlaire, and J. P. Thiran. Behavioral priors for detection and tracking of pedestrians in video sequences. International Journal of Computer Vision, 69(2):159-180, 2006

[10] B. Zhou, X. Wang, and X. Tang. Understanding collective crowd behaviors: Learning a mixture model of dynamic pedestrian-agents. In Proc. of CVPR. IEEE, 2012.

[11] X. Wang, X. Ma, and W. E. L. Grimson. Unsupervised activity perception in crowded and complicated scenes using hierarchical bayesian models. IEEE Transactions on Pattern Analysis and Machine Intelligence, 31(3):539-555, 2009.

[12] B. Zhou, X. Tang, and X. Wang. Measuring crowd collectiveness. In Proc. CVPR, IEEE, 2013.

[13] B. Zhou, X. Tang, H. Zhang, and X. Wang. Measuring crowd collectiveness. IEEE Transactions on Pattern Analysis and Machine Intelligence, 36(8):1586-1599, 2014.

[14] R. Mehran, A. Oyama, and M. Shah. Abnormal crowd behavior detection using social force model. In Proc. of CVPR. IEEE, 2009.

[15] M. Moussa"1d, D. Helbing, and G. Theraulaz. How simple rules determine pedestrian behavior and crowd disasters. Proceedings of the National Academy of Sciences, 108(17):6884-6888, 2011. 